\title{
Distinct Roles of the Rho-GTPase Associated Kinases PAK and ROCK in the Regulation of Dendritic Spines and Synaptic Plasticity
}

\author{
Suhail Asrar ${ }^{1,2}$ and Zhengping Jia*,1,2 \\ ${ }^{l}$ Neurosciences and Mental Health, Hospital for Sick Children, Toronto, ON, Canada \\ ${ }^{2}$ Department of Physiology, University of Toronto, Toronto, ON, Canada
}

\begin{abstract}
Dendritic spines are highly specialized neuronal structures that are the major postsynaptic sites for excitatory input. These actin-rich expansions are highly versatile in adapting their morphology and density towards the support of synaptic transmission and plasticity. Among the chief factors known to be crucial in the modulation of the actin cytoskeleton, the Rho-GTPases and their associated signaling effectors are particularly important. This signaling system is involved in numerous regulatory processes, including cell morphology, structural dynamics and cell motility. Accordingly, the disruption of Rho-related signaling has a profound effect on the integrity of neurons, resulting in abnormalities with neurite outgrowth, dendritic arborization, spine properties and plasticity. These perturbations can dramatically alter normal synaptic function, including hippocampal long-term potentiation (LTP), resulting in cognitive defects. Additionally, RhoGTPase-associated signaling disorders have also been implicated in numerous forms of mental retardation. Therefore, the elucidation of the underlying mechanisms involved in this pathway and their critical association with dendritic spines remains a major focus of research concerning the cellular basis of cognitive function. Here we will discuss our recent data obtained utilizing knockout animals deficient in the expression of PAKs (p21-activated kinases) and ROCKs (Rhokinases), predominant protein kinases known to be directly activated by the Rho-GTPases. A downstream target for both PAKs and ROCKs, LIMKs (Lin-11, Isl-1, and Mec-3 kinase), will also be discussed. While it is evident that these kinase families all serve towards spine and synaptic regulation, their individual roles in the achievement of this goal may be quite different.
\end{abstract}

\section{INTRODUCTION}

The presence of minute membranous extensions on the surface of neuronal dendrites has been a cornerstone of central nervous system research for many decades. Ironically, when Cajal first discovered dendritic spines in 1888, many of his scientific peers dismissed them as an aftereffect of the Golgi method he was utilizing, and it was a further eight years before he was able to provide more authoritative evidence regarding their existence using Methylene Blue [1]. Since then, modern exploratory techniques such as microscopy and electrophysiology have allowed a more thorough examination of the structure and mechanisms underlying these expansions. Dendritic spines are thought to be reservoirs of signaling molecules that facilitate synaptic strengthening, and are extremely versatile in their ability to alter their volume and shape via actin remodeling upon requirement. Hence, the regulation of these protrusions is of paramount importance in relation to proper neuronal function and synaptic plasticity [2,3]. Furthermore, many forms of mental retardation are linked to perturbations in dendritic spine morphology [4; see other reviews in this volume]. In the search for important administrators of the cytoskeleton, RhoGTPases and their associated protein kinases have emerged as crucial players in spine actin regulation. These GTPases,

*Address correspondence to this author at the Neurosciences and Mental Health Program, Hospital for Sick Children, Department of Physiology, University of Toronto, Toronto, Ontario, Canada;

E-mail: zhengping.jia@sickkids.ca including Rho, Rac and Cdc42, are guanine binding proteins that largely alternate between activated GTP-bound states and inactivated GDP-bound states. The transition of certain types of Rho-GTPases between these states is coordinated by the guanine nucleotide exchange factors (GEFs), GTPase activating or accelerating proteins (GAPs), and the guanine nucleotide dissociation inhibitors (GDIs). A smaller group of Rho-GTPases do not undergo the cycling states described above, and are mostly GTP-bound. These are described as atypical Rho-GTPases, and include Rnd, Rho H, Rho U and Rho V [5].

Manipulations in the Rho-GTPase signaling pathways can lead to a large number of deficits in spine morphology [6-9], the structural remodeling of neurons [10-12], synaptic plasticity [13-15], as well as changes in response to specific learning tasks [16-18]. Additionally, Rho-associated kinases are involved in both syndromic and non-syndromic mental retardation [19]. Therefore, delving further into the relationship between the components of Rho-GTPase signaling and dendritic spine morphology and function is of considerable interest. In this review, the roles of PAKs (p21-activated kinase), ROCKs (Rho-kinase) and their downstream target LIMKs (Lin-11, Isl-1, and Mec-3 kinase) will be specifically addressed. Although these kinases are also involved in other aspects of brain development and function [20-26], we will specifically focus on spine and synaptic regulation in the adult. 


\section{PAKS (P21-ACTIVATED KINASES)}

The PAK family of serine/threonine kinases consists of six isoforms in humans, and were the first Rho-associated kinases to be discovered [27]. Each of these isoforms contains an $\mathrm{N}$-terminal regulatory domain and a $\mathrm{C}$-terminal catalytic domain. Based on their structural similarities, the PAK isoforms are further subdivided into two groups. Group I comprises of PAK1-3, while Group II includes PAK 4-6 [20]. The major distinction between the two groups is in their regulatory domain. The Group I PAK isoforms are limited in their function by an auto-inhibitory switch domain (AID). The inhibitory effect of this domain is disrupted by the interaction of active Rho-GTPases with the PBD (p21Rho-binding domain), leading to kinase activation. In contrast, Group II PAKs do not undergo AID-dependent regulation because its members are missing the AID motif. In spite of their structural differences, both groups of PAK are widespread in the CNS [21-23]. PAKs interact with members of the Rac/Cdc 42 family, including Rac3, RhoV, RhoU and RhoQ. Other studies have also revealed the ability of PAKs to be activated through GTPase-independent mechanisms through the actions of diverse molecules [28] such as sphingosine (a cell membrane lipid), PIX (a Rac GTP exchange factor), and Filamin A (a cytoplasmic protein that crosslinks cortical actin).

\section{GROUP I PAKS AND SYNAPTIC FUNCTION}

A vast number of studies concerning spines and synapses have been focused on Group I PAKs. Although a large number of downstream signaling targets have been identified in cultured cell lines [28], MLCK (myosin light-chain kinase) and LIMK are of particular interest as the inhibition of the former and the stimulation of the latter both lead to an increase in actin polymerization and the formation of dendritic spines [19,29-31]. Group I PAKs have also been implicated in important activities such as cellular motility, morphology, apoptosis signaling and gene transcription. PAK2 is widely expressed in most regions in the body, whereas PAK1 and PAK3 are more prominent in the brain [17,32-35], including hippocampal dendritic spines [36-38]. Both PAK1 and PAK3 are present in neurons at various stages of development in rodents, being more prominent in the hippocampus and cortex [32,39-41]. These results suggest that, in addition to adult function, PAK1/3 may also play a role in brain development. An early study utilizing transgenic mice took advantage of the ability of AID in inhibiting the catalytic activity of PAKs in order to inhibit the function of these kinases [42]. This study revealed a lessening in the number of spines at the level of the cortex, in addition to a general shift towards spines that were larger in size. Synaptic transmission was enhanced in cortical neurons, with an augmentation of long-term potentiation (LTP) as well as a diminishment of long-term depression (LTD) in comparison to wild-type controls. In contrast, these transgenic mice displayed no demonstrable differences in either structure or synaptic plasticity in the hippocampal region. The authors attributed this disparity to the fact that significant levels of activated PAK still existed in the hippocampus of these animals, a factor which may have contributed to the lack of a visible phenotype in this area. While this study provided important information on the general role of PAKs in the cortex, it did not explore the specific functions of the indi- vidual members of the Group I family. Furthermore, the incomplete blockage of PAK activity in the hippocampus prevented a thorough analysis of PAKs function in this important brain region. Another study extended these findings to implicate PAK phosphorylation of myosin II regulatory light chain (MLC) in the promotion of spine stability [43]. The expression of dominant-negative PAK1 and PAK3 constructs in hippocampal neurons revealed that the suppression of the kinase activity of either of these proteins interfered with spine morphogenesis and formation. However, it was unclear whether these constructs were specific in inhibiting the actions of PAK1 and PAK3 respectively without affecting other members of the Group I PAKs.

\section{PAK1 AND PAK3 KNOCKOUT STUDIES}

The utilization of knockout mice deficient in the expression of PAK1 or PAK3 enabled a detailed investigation of each of these kinases especially with regards to their effects on dendritic spines and synaptic plasticity [17,35]. The PAK1 knockout mice displayed no detectable PAK1 protein, while not affecting the levels of its group members PAK2 or PAK3. General behavior was also unaltered in these mice, with no changes in fertility, viability, lifespan and homecage locomotor activities. Fixed brain sections obtained from these mutants revealed no abnormalities in the gross anatomy of the CNS. Electron micrography of brain sections as well as immunostaining of cultured hippocampal neurons also revealed spines and synapses with no structural deficits in PAK1 knockouts. These results suggest that the role of PAK1 in the development of the brain or formation of the synapse may not be essential, potentially due to functional overlap with other Group I kinases. Accordingly, PAK1 knockout mice showed no significant deficits in basal excitatory synaptic transmission or paired-pulse facilitation (PPF), a short-term form of plasticity that is indicative of presynaptic changes. However, in contrast to the morphological results, PAK1 knockout mice displayed a profound deficiency in synaptic plasticity. Long-term potentiation (LTP) is the most widely studied form of plasticity thought to be the molecular correlate for learning and memory $[13,44]$. Induction of the late-phase of LTP (considered to correspond to longterm memory) in the CA1 region of the hippocampus induced by using multiple trains of high-frequency stimulation (HFS) revealed a severe diminution of potentiation in brain slices from mutant mice in comparison to the wild-type, whereas early-phase LTP induced by one or two trains of HFS was unaffected. This selective reduction of the longlasting plasticity in PAK1 knockouts suggested that PAK1 is only required for certain forms of plasticity through mechanisms that are distinct from the involvement of spine morphology. An interesting finding in cultured hippocampal neurons obtained from PAK1 knockout mice was that both F-actin and the NMDA-induced activation of the actinbinding protein cofilin were significantly reduced at the level of the dendritic spines. In this regard, it has previously been suggested that actin can potentially regulate plasticity through mechanisms independent of their structural role $[45,46]$. Actin polymerization may also be involved in processes that lead to the synthesis of the protein kinase PKMzeta [47], a key protein required for the maintenance of the late-phase of LTP [48]. Additionally, PAKs have been shown to activate the MAPK signaling cascade through the 
direct phosphorylation of the upstream components MEK (mitogen-activated protein kinase kinase) and Raf- 1 and on Ser298 [49] and Ser 338 [50] respectively. The MAPK pathway is known to be a major player in both proteinsynthesis dependent $[51,52]$ and protein-synthesis independent [53] forms of LTP, and PAKs may potentially play a role in the activation of this cascade during synaptic plasticity.

In addition to PAK1, genetic investigations were also performed on PAK3 [17]. PAK3 possesses a high degree of homology with PAK1, sharing around $90 \%$ similarity in both the N-terminal PBD and C-terminal catalytic domains [21]. Importantly, PAK3 is one of the genes identified in X chromosome-linked non-syndromic mental retardation or MRX $[54,55]$. In comparison to PAK1, PAK3 is present more exclusively in neurons, suggesting a particular importance of this isoform in the CNS. Early studies utilizing antisense and RNA interference to inhibit PAK3 activity revealed abnormalities in spine formation, with increased levels of filopodia-like protrusions and immature spines in rat organotypic slice cultures [56]. These morphological changes were accompanied by differences in the level of AMPA receptor expression at the synapse, as well as reduced LTP when induced through a pairing protocol. In another study, PAK3 function in regards to spine formation was found to be significantly more associated with Cdc42 than Rac1 [57]. Consistent with these in vitro studies, hippocampal LTP in PAK3 knockout mice [17] was also found to be considerably hampered in comparison to controls, in spite of apparently normal basal synaptic strength and presynaptic function. However, the presentation in knockout mice differed from in vitro studies in that the PAK3 mutants did not display any significant alterations in spine morphology or density in both cultured neurons and fixed brain sections. Additionally, despite past evidence stressing the importance of PAKs in the regulation of the actin cytoskeleton, PAK3 mutants did not display any detectable alterations in F-actin in the spine of cultured hippocampal neurons. This was in clear contrast to the obvious reduction in the amount of F-actin by its closely associated family member PAK1. Additionally, PAK3 has also not been demonstrated to activate LIMKs, unlike PAK1. Therefore, actin-independent mechanisms had to be explored in order to explain the deficient LTP seen in PAK3 knockout mice. In this regard, the role of the transcription factor CREB (cAMP response element binding) was evaluated. Numerous studies have revealed CREB to be crucial to both LTP as well as long-term memory [58-60]. The assessment of phosphorylated or active CREB Ser-133 in PAK3 mutant mice revealed a significant reduction of this factor in comparison to wild-type controls. Therefore, PAK3 may be involved in the regulation of CREB activity. Interestingly, the level of activated CREB was unaffected in PAK1 knockout mice [35], indicating the specificity of PAK3 in the regulation of this transcription factor. Surprisingly, the decrease in CREB function and synaptic plasticity in mutants was not accompanied by deficits in spatial learning in the Morris Water Maze (MWM) test. Instead, PAK3 knockouts displayed an accelerated extinction in the conditioned taste aversion (CTA) test. The dependence of the former test on the hippocampus in contrast to the latter may suggest that PAK3 may affect behavior that is more reliant on the function of the amygdala and cortex. However, it has been noted that knockout animals with altered LTP may reveal deficien- cies in certain types of hippocampal-dependent behavioral paradigms in comparison to others [61,62], suggesting that additional tests may be required in PAK3 knockout mice. Furthermore, instances where CREB depletion alters hippocampal-independent CTA tests while not affecting hippocampal-dependent tasks have been observed previously $[63,64]$.

Taken together, the above results provide considerable evidence to support the role of both PAK1 and PAK3 in the regulation of dendritic spine function and synaptic plasticity (Fig. 1).

\section{ROCKS (RHO-KINASES)}

The most extensively known target of RhoA-GTPases are the Rho-associated protein kinases or ROCKs $[65,66]$, which are members of the diverse AGC (cAMP-dependent, cGMPdependent and protein kinase C) family of protein kinases. These proteins have been implicated in a number of vital cellular processes, including smooth muscle contraction, adhesion, survival and synaptic remodeling. They consist of a kinase domain, a coiled-coil region with a RBD (RhoAbinding domain), and a carboxyl-terminal domain. ROCK function can be inhibited through antagonists (such as Y27632 and fasudil) that interact with the kinase domain. ROCKs comprise two closely related isoforms, ROCK1 and ROCK2, the latter of which is abundantly expressed in the brain and therefore may be more specifically involved in spine and synaptic regulation. In addition to RhoA, ROCK2 in particular is known to be regulated by a variety of factors [24], including arachidonic acid [67], the sphingolipid sphingosylphosphorylcholine [68], and phosphoinositide 3kinase (PI3K) [69]. In terms of cytoskeletal regulation, ROCK2 acts on a large number of downstream effectors, including myosin-II regulatory light chain phosphatase (MLCP) [25], $\alpha$-Adducin [70], and LIMK [71]. The activation of MLCKs and myosin by ROCKs can lead to retraction of both axonal and dendritic growth cones through actindependent mechanisms $[29,30]$, which might be relevant to spine shape changes.

The investigation of ROCK function in relation to dendritic spines and synaptic plasticity has been largely dependent on two types of studies, one pharmacological and the other genetic. In one study using cultured mouse hippocampal slices, neurons treated with the ROCK inhibitor Y-27632 were found to have longer spines without any changes in the size of the spine heads [72]. To confirm whether the administration of Y-27632 caused the formation of new spines, or alternatively affected spines that were already present, the authors imaged spines prior to and following the addition of the drug. The increased mean length of spines was largely due to an increase in the number of recently formed protrusions. Furthermore, shorter-term incubation with Y-27632 (10 minutes to 2 hours) resulted in a temporary augmentation of the protrusive ability of a subset of spines lasting 24 hours. Therefore, these results suggested that ROCKs are also directly involved in the formation of spines as well as the maintenance of their morphology. In accordance with these morphological studies, the administration of Y-27632 was also shown to enhance the magnitude of LTP in acute hippocampal slices [73], suggesting that ROCK may play an inhibitory role in LTP. However, a recent study using the 


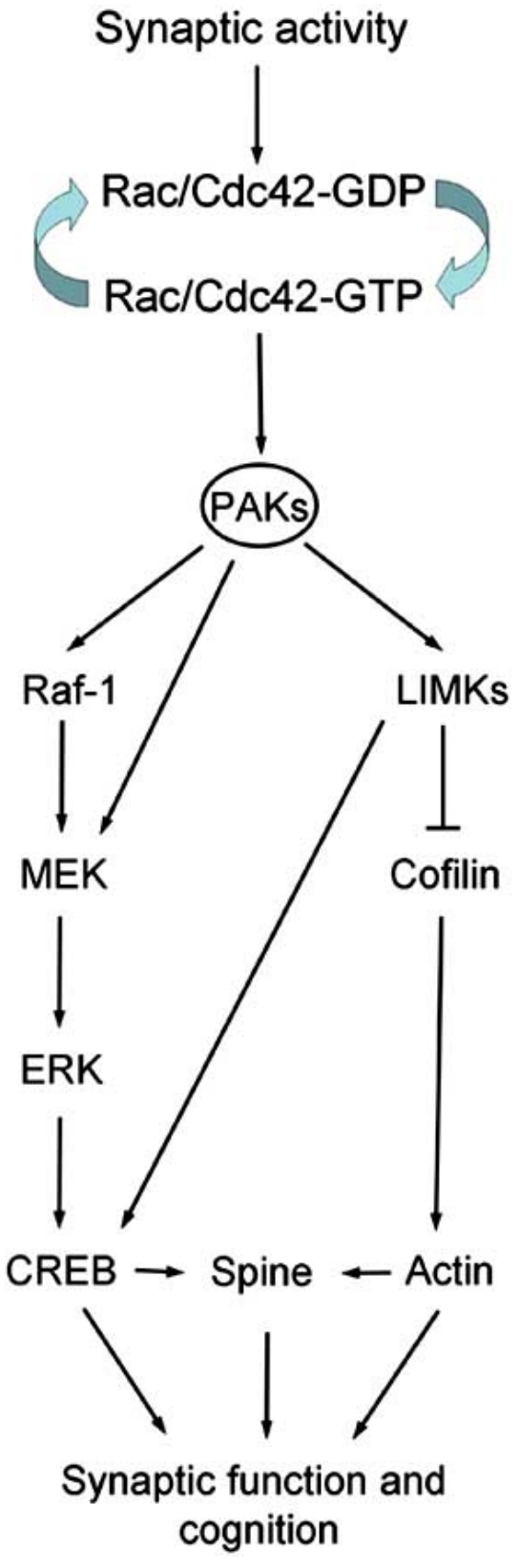

Fig. (1). PAKs regulate CREB and stimulus-dependent cofilin activity. Activation of PAKs by Rac/Cdc42 leads to the stimulation of both Raf-1 and MEK to facilitate the Raf-1-MEK-ERK signaling cascade, potentially to activate the transcription factor CREB. Additionally, some PAKs can modulate actin through the actions of LIMKs. Cofilin activity in this PAK-dependent pathway is activityreliant, requiring the stimulation of synaptic receptors. These pathways help maintain normal spine formation and synaptic activity, such the occurrence of hippocampal LTP. PAK $=$ p21-activated kinase, $\mathrm{CREB}=\mathrm{cAMP}$ response element binding, $\mathrm{MEK}=$ mitogenactivated protein kinase kinase, MLCK $=$ myosin light-chain kinase, LIMK = Lin-11, Isl-1, and Mec-3 kinase, LTP = Long-term potentiation.

more specific ROCK inhibitor H-1152 revealed that the presence of this drug reduced the amplitude of potentiation [74]. Importantly, an involvement of ROCK signaling in long-term fear memory and anxiety-related activities has also been demonstrated $[75,76]$.

\section{ROCK2 KNOCKOUT STUDIES}

Since the use of ROCK inhibitors could not distinguish different members of the ROCK family, we used genetic manipulation to specifically ablate ROCK2, the predominant brain isoform [77]. These mice exhibited no appreciable differences in the gross anatomy of the CNS, including the hippocampus. Electrophysiological studies revealed the importance of ROCK2 towards the maintenance of basal synaptic strength, with fEPSPs being considerably reduced over a range of stimulation intensities in the knockout mice in comparison to wild-type littermate controls. Further, the induction of hippocampal late-phase LTP was also significantly attenuated in mutant mice in the absence of changes in NMDA or AMPA receptor channel properties. These genetic results on synaptic potentiation were consistent with the effect of the ROCK inhibitor H-1152, but in odds with that of Y-27632. These differences may be explained by the nonselective actions of Y-27632 on additional members of the large AGC family of serine-threonine kinases other than ROCKs. Electron microscopic studies in ROCK2 mutants revealed a reduction in synaptic density at CA1 synapses, a factor which could possibly account for the decrease in basal synaptic strength. This was corroborated by immunohistochemical staining using fixed hippocampal sections, where the diminished co-localization of presynaptic marker synapsin and vesicular glutamate transporter 1 or vGLUT1 was also seen. Additionally, cultured hippocampal neurons obtained from ROCK2 knockout mice revealed an increase in the average spine length, with a larger number of spines appearing as filopodia-like protrusions that are characteristic of immaturity. Since the actin cytoskeleton has previously been demonstrated as an important target of Rho/ROCK signaling [78], possible abnormalities in this region where also examined in mutants. Cultured neurons revealed a deficit in the fluorescence intensity ratio of spine/dendrite phalloidin staining, confirming the importance of ROCK2 in the regulation of spine F-actin. Interestingly, the basal phosphorylation state of cofilin is reduced in ROCK2 knockout mice but there is no change in the NMDA-dependent cofilin activation. Therefore, these results emphasized the importance of ROCK2 in the regulation of spine activity through cofilin/actin-dependent mechanisms that are also essential for maintaining morphology (Fig. 2).

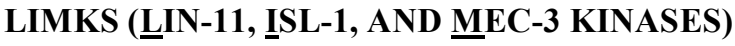

A common substrate for PAKs (PAK1 and PAK4) and ROCKs is the LIM family of kinases. Therefore, the differential regulation of LIMKs by either PAKs or ROCKs may represent a key component of these two pathways in the modulation of dendritic spine function and synaptic plasticity. LIMKs consist of two members, LIMK1 and LIMK2, the former of which has been largely studied in its relationship to the CNS. LIMKs contain two N-terminal LIM domains, a PDZ domain, and a C-terminal kinase domain. These kinases provide a crucial pathway for the promotion of actin reorganization through phosphorylation and inactivation of ADF/cofilin (actin depolymerization factors) [79,80]. LIMKs can be potently activated through direct phosphorylation at Thr508 by both PAKs and ROCKs, although other kinases, including PKA (protein kinase A) and phosphorylation-independent mechanisms [81] may also play an important regulatory role. LIMKs can also be additionally regu- 


\section{Synaptic activity}
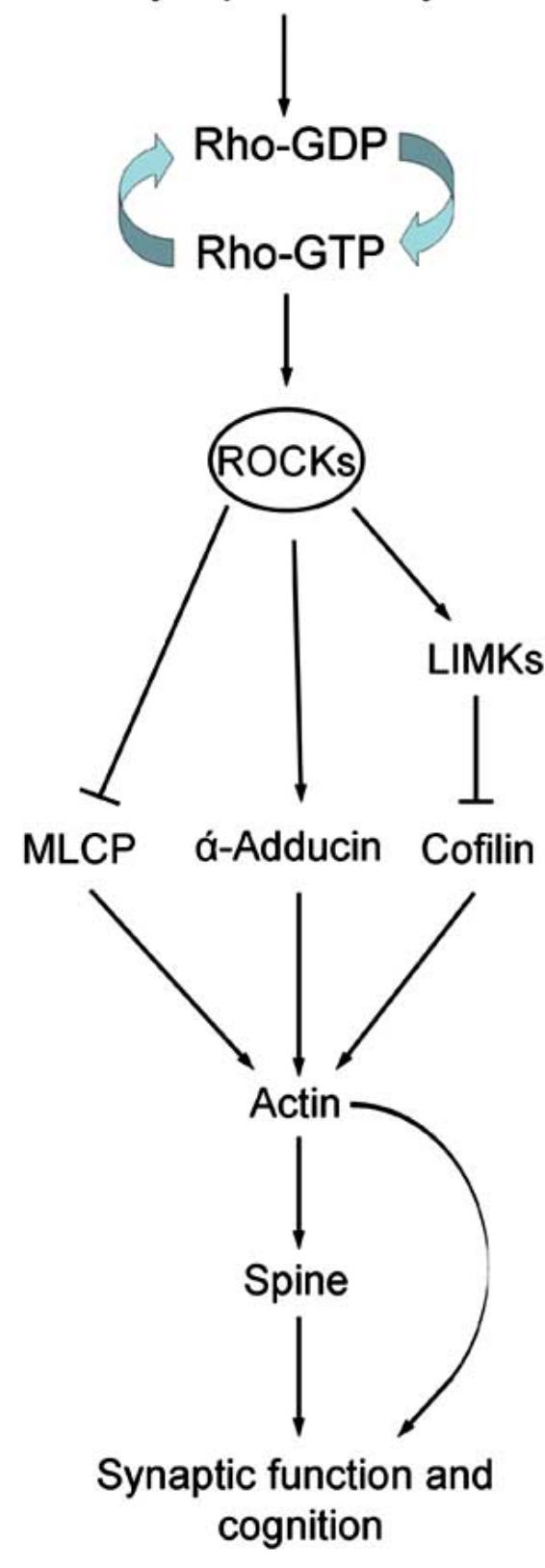

Fig. (2). Actin regulation and basal cofilin phosphorylation is dependent on ROCKs. The stimulation of ROCK activity by Rho is crucial for the modulation of actin through the actions of the downstream substrates MLCP, $\dot{\alpha}$-Adducin and LIMKs. ROCK-dependent cofilin regulation is activity-independent, and only occurs at the basal level with no effect under NMDA-stimulated conditions. This facilitation of actin dynamics by ROCKs is vital for the maintenance of spine morphology, and thus synaptic plasticity and cognition. ROCK $=$ Rho-kinase, MLCP $=$ myosin-II regulatory light chain phosphatase, LIMK = Lin-11, Isl-1, and Mec-3 kinase.

lated by a number of varied factors [26] such as transforming growth factor-beta 1 [82], bone morphogenetic protein [83], slingshot [84] and Rnf6 [85]. An interesting study also implicated a specific microRNA (miR-134) in the inhibition of LIMK1 translation, a process that was reversed by the administration of brain-derived neurotrophic factor [86].

\section{LIMK1 AND LIMK2 KNOCKOUT STUDIES}

To specifically address the role of LIMK1 in the brain, knockout mice were created by homologous recombination [87]. These mutants showed no detectable levels of LIMK1 mRNA or protein, while expressing normal levels of LIMK2, PAKs, ROCK2 and cofilin. LIMK1 knockout mice revealed no abnormalities in the gross structure of brain, including regions where they are highly expressed such as the hippocampus, the cortex and the olfactory bulb. Western blot analysis was performed on samples prepared from whole-brain and hippocampal slices to examine the phosphorylation status of $\mathrm{ADF} /$ cofilin. The results indicated that phosphorylated (inactive) cofilin was significantly reduced in knockout mice, suggesting a critical role for LIMK1 in the phosphorylation and inhibition of basal ADF/cofilin activity. In addition, the stimulus-dependent regulation of cofilin in response to external stimuli, including activation of glutamate receptors, was also impaired in LIMK1 knockout brain slices. These data provided strong evidence that LIMK1 is important for both the constitutive and activity-dependent regulation of ADF/cofilin phosphorylation/dephophorylation. Additional studies were also performed to assess if there was any abnormalities at the level of the actin cytoskeleton. Immunostained cultured hippocampal neurons revealed that growth cones were absent or diminished in size in knockout neurons, along with anomalous clustering of both cofilin and actin in comparison to wild-type animals. Phalloidin staining was also observed to be of lower intensity and more uniformly spread between the spine head and adjacent dendritic areas in mutant neurons, whereas the former region was more intensely stained in wild-type controls. These results demonstrated that LIMK1 is crucial for the accumulation and distribution of F-actin in the dendritic spine. Consistent with the molecular deficits, LIMK1 mutant neurons displayed abnormalities in spine morphology. While the majority of spines in the wild-type animals are mushroom-shaped with thin necks and large heads, the LIMK1 mutants exhibited thick necks and small heads, characteristic of immature or developing neurons. Electron microscopy of hippocampal CA1 areas also demonstrated that the size of the postsynaptic density (PSD) of mutant synapses was also considerably decreased. Therefore, these results ascertained the importance of LIMK1 in the preservation of normal spine and synaptic morphology in both in vivo and culture models. To explore the impact of these alterations to the actin cytoskeleton and dendritic spines, electrophysiology in the hippocampal CA1 region was also performed. Surprisingly, the early-phase LTP induced by HFS $(50$ or $100 \mathrm{~Hz})$ was found to be significantly enhanced in knockout mice in comparison to controls. Interestingly, the presence of low concentrations of the F-actin inhibitors cytochalasin D and Latrunculin B significantly enhanced LTP in wild-type animals while not affecting the mutants, suggesting that the heightened plasticity in the absence of LIMK1 is mediated through actin dynamics. More recent studies indicate that although early-phase LTP is enhanced in LIMK1 knockout mice, the late-phase of LTP is conversely abolished (unpublished data). Together, these results suggest that LIMK1 differentially regulates the early- and late-phases of LTP, possibly through distinct mechanisms. The role of LIMK1 in the modulation of the actin cytoskeleton was extended to presynaptic activities, with mutants demonstrating a faster and 
Table 1. Summary of Rho-GTPase Associated Kinase Studies in Knockout Animals

\begin{tabular}{|c|c|c|c|}
\hline Protein & Functional Roles & $\begin{array}{l}\text { Spine Alterations in Knockout } \\
\text { Mice }\end{array}$ & Other Manifestations \\
\hline PAK1 & Effector of Rac and Cdc42. & $\begin{array}{l}\text { Normal spine and synaptic mor- } \\
\text { phology. Deficiency in actin accu- } \\
\text { mulation and NMDA-induced acti- } \\
\text { vation of cofilin. }\end{array}$ & Significantly reduced LTP. \\
\hline ROCK2 & Main target of RhoA-GTPases. & $\begin{array}{l}\text { Increase in filopodium-like struc- } \\
\text { tures and average spine length. } \\
\text { Abnormalities in actin accumulation } \\
\text { and basal phosphorylation of } \\
\text { cofilin. }\end{array}$ & $\begin{array}{l}\text { fEPSPs are diminished over a range } \\
\text { of stimulus intensities. LTP is sig- } \\
\text { nificantly attenuated. Reduction of } \\
\text { synaptic density in CA1 synapes, } \\
\text { with decreased co-localization of } \\
\text { synapsin and vGLUT1. }\end{array}$ \\
\hline
\end{tabular}

Abbreviations: PAK (p21-activated kinase), ROCK (Rho-kinase), LIMK (Lin-11, Isl-1, and Mec-3 kinase), NMDA (N-methyl-D-aspartic acid), CREB (cAMP response element binding), CTA (conditioned taste aversion), LTP (long-term potentiation), vGLUT1 (vesicular glutamate transporter 1).

larger degree of synaptic depression as well as an increased rate of mEPSCs (miniature excitatory postsynaptic currents). In accordance with the morphological and electrophysiological changes, LIMK1 knockout mice exhibited significant abnormalities in behavioral responses, including increased locomotor activity and impairments in learning and memory. Of particular interest is that LIMK1 knockouts showed significant deficits in spatial learning in the the water maze test; visuospatial deficits and hyperactivity are hallmarks of human Williams syndrome, a developmental disorder where several proteins (including LIMK1) are abnormally expressed. The exploration of possible abnormalities in the dendritic spine morphology (such as those seen in LIMK1 mutant mice) of these patients may shed new light towards the better understanding of this disorder.

In addition to LIMK1, knockout mice devoid in LIMK2 were developed as well. However, analyses of these mutants did not reveal strong phenotypical differences in either spine morphology or synaptic physiology between wild-type and LIMK2 knockout animals, although double knockout mice lacking both LIMK1 and LIMK2 showed more severe deficits in the level of phosphorylated cofilin [88]. More studies are clearly needed to further analyze LIMK2 and LIMK1/2 double mutants to determine their relative contribution to spine and synaptic regulation.

\section{MAKING SENSE OF KINASES IN REGULATION OF SPINES}

Although the importance of PAKs, ROCKs and LIMKs in regulating the actin cytoskeleton, the dendritic spine and synaptic function is widely accepted, the precise roles of these kinase families and their individual members are only beginning to be understood (Table 1). While both PAK1 and PAK3 knockout mice showed an impairment in LTP, the relative causes for these deficiencies were surprisingly different. PAK1 knockouts displayed abnormalities in both basal actin regulation and stimulus-dependent cofilin activity, suggesting a more prominent role for actin-based processes in synaptic regulation. Interestingly, despite changes in spine F-actin and cofilin, neither spine morphology nor spine density was disrupted by PAK1 deletion. On the other hand, PAK3 mutant studies reflected no significant changes in spine F-actin or its regulatory proteins (including cofilin), and consequently no changes in the spine structure. These results may be reflective of the inability to demonstrate that PAK3 can specifically activate LIMKs thus far. Instead, PAK3 knockouts displayed reduced CREB activity, implying that abnormal regulation of CREB-dependent mechanisms may contribute to the PAK3 knockout deficits. Therefore, despite the large structural and biochemical similarities between PAK1 and PAK3, these genetic studies have revealed important in vivo functional differences and distinctions in their underlying molecular signaling. The reasons for such differential effects are unknown, but may be related to their relative localization. PAK1 is present mainly in the apical dendrites and spines, whereas PAK3 is mostly within the cell body and proximal dendrites [42,89]. Importantly, both actin and CREB have been implicated as crucial factors during the occurrence of the late-phase of LTP, a phenomenon which is deficient in both PAK1 and PAK3 knockout mice. However, actin dynamics are also critically involved in receptor trafficking at the synapse [90], a process key to the early-phase of LTP and other forms of long lasting synaptic plasticity. Therefore, it is important to investigate these additional forms of plasticity in PAK1 and PAK3 knockout mice, and to determine the relative importance and underlying mechanisms of actin and CREB in mediating PAK actions. In addition to the activation of LIMKs (and thus inhibition of 
cofilin) to regulate actin dynamics, there are a number of PAK-related signaling pathways involved in cytoskeletal regulation [22,23], but the significance of these pathways in mediating the action of PAKs in context of spine and synaptic function is unknown. With respect to CREB regulation by PAK3, at least two potential mechanisms exist. The first is through LIMK1, which in some cell types can directly phosphorylate CREB at Ser133, while the second is through the activation of the MAPK cascade, a known stimulator of CREB activity [91]. PAKs can also directly phosphorylate and activate Raf and MEK, upstream regulators in the MAPK signaling cascade. The significance of each of these two pathways in mediating PAK3 action on CREB and synaptic plasticity warrants further study.

In comparison to earlier studies utilizing small inhibitory RNA or dominant-negative constructs to demonstrate that both PAK1 and PAK3 are important for spine morphology and synaptic plasticity $[42,43]$, the lack of morphological changes in dendritic spines in either PAK1 or PAK3 knockout mice was unexpected. It is possible that subtle structural/morphological changes may have escaped detection through the techniques utilized. However, there are several possibilities to explain these discrepancies, including differences in experimental conditions and chronic versus acute inhibition. It is also likely that dominant-negative constructs produced more dramatic effects on spine morphology than individual gene deletions because these constructs affect more than one member of the PAK family. This suggests that PAK1 and PAK3 may have redundant function in relation to morphological regulation, and spine deficits may only arise if both PAK1 and PAK3 activity is subdued. Therefore, it would be important to analyze double or triple knockout mice lacking combinations of PAK family members. Inducible or regional disruptions of PAKs would also be useful to address potential developmental compensations that may be associated with global genetic deletions.

In addition to PAK1, ROCK2 knockout mice also displayed abnormalities in both cofilin and actin regulation. However, only the basal phosphorylation of cofilin (and thus actin) was selectively subdued in ROCK2 mutants. This is in contrast to PAK1 knockout mice, where only NMDAdependent cofilin regulation was specifically affected. Similarly, the alterations in dendritic spine morphology and basal synaptic response were only seen in ROCK2, but not in PAK1 mutant mice. These results suggest that ROCK2 and PAK1 play distinct roles in spine and synaptic regulation by regulating basal and activity-dependent cofilin actions respectively, potentially through differential regulation of their downstream substrate LIMK or via a cofilin phosphatase (see review by Pontrello and Ethell in this volume). In support of this notion, a recent study implicated the activity of ROCK and LIMK1 in an Ephrin B receptor-FAK (focal adhesion kinase) mediated cascade that was crucial for the maintenance of normal physiological integrity and shape of dendritic spines through cofilin regulation [92]. The selective deficits in hippocampal LTP without changes in spine properties and basal synaptic function in PAK3 knockout mice are also consistent with the specific role of PAKs in activitydependent neuronal function. Since LIMK is a common substrate to both PAKs and ROCKs, it is not surprising to observe the vast array of deficits seen in LIMK1 mutant animals. However, the changes in synaptic plasticity in LIMK1 knockouts are clearly different from those viewed in both PAK and ROCK2 mutants, indicating that co-coordinated regulation of LIMKs by PAKs and ROCK2 is critical for normal synaptic function. The precise mechanisms underlying this complex regulation remain to be elucidated. Additional experimentation with PAK/ROCK double knockout mice may provide valuable information with regards to interactions between these kinase families. Moreover, the suggested relationship between LIMKs and the transcription factor CREB [93] merits further attention as a vital mechanism in the establishment of synaptic plasticity and memory, particularly through PAK-dependent regulation.

\section{CONCLUDING REMARKS}

Taken together, the above genetic studies have clearly demonstrated the distinct and critical roles played by the Rho-GTPase-activated protein kinases PAK1/3 and ROCK2 in the regulation of spine properties and synaptic function. In particular, the roles of these protein kinases in mental retardation and other disorders have provided increased relevance and importance towards their continued study. Since most of the presented data have been obtained using fixed brain tissues or cultured neurons, the roles of these kinases in spine plasticity in live neurons remains an exciting area to explore. Additionally, the manner in which these kinases are specifically activated by neuronal or synaptic activities and their relation to synaptic receptors and associated proteins are also important venues of investigation. The localization of these active kinases has also been of significant interest in recent years [42]. In this regard, the use of a phospho-specific antibody revealed that activated PAK co-localized with PSD-95, suggesting local activation at the level of the synapse [43]. Another study demonstrated that the hyperactivation of PAK1 in neurites led to changes in neuronal morphology as well as the inability to distinguish between axons and dendrites [94]. Therefore, the temporal and spatial restrictions of activated PAK may also play a role in the functional differences observed in mutant animals. The impact of PAKs, ROCKs and LIMKs in brain regions other than the hippocampus as well as during different stages of development also needs to be further elaborated. The possibility that redundant function or mutual compensation may have prevented phenotypic expression among the members of the PAK and ROCK families in single knockout animals could potentially be addressed through the use of double or triple knockout mice. The use of genetically altered mice in combination with other molecular manipulations such as more specific functional probes and RNA interference may prove beneficial and necessary towards achieving these studies. In addition, the vast array of substrates targeted by these kinases $[21,25]$ requires careful evaluation to precisely elucidate the functions they effect. It is our hope that the further elaboration of these crucial kinase families will contribute to an understanding of the molecular mechanisms underlying spine and synaptic function, and provide potential therapeutic targets to treat related mental and neurological disorders.

\section{ACKNOWLEDGEMENTS}

This work was supported by grants from the Canadian Institutes of Health Research (CIHR MOP-42396) and Ontario Mental Health Foundation. Suhail Asrar was supported by the Ontario Graduate Scholarship (OGS). 


\section{REFERENCES}

[1] García-López P, García-Marín V, Freire M. The discovery of dendritic spines by Cajal in 1888 and its relevance in the present neuroscience. Prog Neurobiol 2007; 83: 110-30.

[2] Sorra KE, Harris KM. Overview on the structure, composition, function, development, and plasticity of hippocampal dendritic spines. Hippocampus 2000; 10: 501-11.

[3] Carlisle HJ, Kennedy MB. Spine architecture and synaptic plasticity. Trends Neurosci 2005; 28: 182-7.

[4] Nadif KN, Van Aelst L. Rho-linked genes and neurological disorders. Pflugers Arch 2008; 455: 787-97.

[5] Aspenström P, Ruusala A, Pacholsky D. Taking Rho-GTPases to the next level: the cellular functions of atypical Rho-GTPases. Exp Cell Res 2007; 313: 3673-9.

[6] Nakayama AY, Harms MB, Luo L. Small gtpases Rac and Rho in the maintenance of dendritic spines and branches in hippocampal pyramidal neurons. J Neurosci 2000; 20: 5329-38.

[7] Tashiro A, Minden A, Yuste R. Regulation of dendritic spine morphology by the Rho family of small GTPases: antagonistic roles of Rac and Rho. Cereb Cortex 2000; 10: 927-38.

[8] Luo L. Rho-GTPases in neuronal morphogenesis. Nat Rev Neurosci $2000 ; 1: 173-80$.

[9] Tada T, Sheng M. Molecular mechanisms of dendritic spine morphogenesis. Curr Opin Neurobiol 2006; 16: 95-101.

[10] Sin WC, Haas K, Ruthazer ES, Cline HT. Dendrite growth increased by visual activity requires NMDA receptor and RhoGTPases. Nature 2002; 419: 475-80.

[11] Li Z, Aizenman CD, Cline HT. Regulation of Rho-GTPases by crosstalk and neuronal activity in vivo. Neuron 2002; 33: 741-50.

[12] Van Aelst L, Cline HT. Rho-GTPases and activity-dependent dendrite development. Curr Opin Neurobiol 2004; 14: 297-304.

[13] Bliss TV, Collingridge GL. A synaptic model of memory: longterm potentiation in the hippocampus. Nature $1993 ; 361: 31-9$.

[14] Malenka RC, Nicoll RA. Long-term potentiation--a decade of progress? Science 1999; 285: 1870-4.

[15] O'Kane EM, Stone TW, Morris BJ. Increased long-term potentiation in the CA1 region of rat hippocampus via modulation of GTPase signalling or inhibition of Rho kinase. Neuropharmacology 2004; 46: 879-87.

[16] Dash PK, Orsi SA, Moody M, Moore AN. A role for hippocampal Rho-ROCK pathway in long-term spatial memory. Biochem Biophys Res Commun 2004; 322: 893-98.

[17] Meng J, Meng Y, Hanna A, Janus C, Jia Z. Abnormal long-lasting synaptic plasticity and cognition in mice lacking the mental retardation gene pak3. J Neurosci 2005; 25: 6641-50.

[18] Diana G, Valentini G, Travaglione S et al. Enhancement of learning and memory after activation of cerebral Rho-GTPases. Proc Natl Acad Sci USA 2007; 104: 636-41.

[19] Ramakers GJA. Rho proteins, mental retardation and the cellular basis of cognition. Trends Neurosci 2002; 25: 191-9.

[20] Eswaran J, Soundararajan M, Kumar R, Knapp S. UnPAKing the class differences among p21-activated kinases. Trends Biochem Sci 2008; 33: 394-403.

[21] Arias-Romero LE, Chernoff J. A tale of two PAKs. Biol Cell 2008; 100: 97-108.

[22] Nikolić M. The PAK1 kinase: an important regulator of neuronal morphology and function in the developing forebrain. Mol Neurobiol 2008; 37: 187-202.

[23] Kreis P, Barnier J. PAK signalling in neuronal physiology. Cell Signal 2009; 21: 384-93.

[24] Shi J, Wei L. Rho kinase in the regulation of cell death and survival. Arch Immunol Ther Exp (Warsz.) 2007; 55: 61-75.

[25] Schmandke A, Schmandke A, Strittmatter SM. ROCK and Rho: biochemistry and neuronal functions of Rho-associated protein kinases. Neuroscientist 2007; 13: 454-69.

[26] Bernard O. LIM kinases, regulators of actin dynamics. Int J Biochem Cell Biol 2007; 39: 1071-6.

[27] Manser E, Leung T, Salihuddin H, Zhao ZS, Lim L. A brain serine/threonine protein kinase activated by $\mathrm{Cdc} 42$ and Rac1. Nature 1994; 367: 40-6.

[28] Bokoch GM. Biology of the p21-activated kinases. Annu Rev Biochem 2003; 72: 743-81.

[29] Govek E, Newey SE, Van Aelst L. The role of the Rho-GTPases in neuronal development. Genes Dev 2005; 19: 1-49.
[30] Linseman DA, Loucks FA. Diverse roles of Rho family GTPases in neuronal development, survival, and death. Front Biosci 2008; 13: $657-76$.

[31] Tanzi RE, Bertram L. Twenty years of the Alzheimer's disease amyloid hypothesis: a genetic perspective. Cell 2005; 120: 545-55.

[32] Manser E, Chong C, Zhao ZS, et al. Molecular cloning of a new member of the p21-Cdc42/Rac-activated kinase (PAK) family. J Biol Chem 1995; 270: 25070-8.

[33] Teo M, Manser E, Lim L. Identification and molecular cloning of a p21Cdc42/Rac1-activated serine/threonine kinase that is rapidly activated by thrombin in platelets. J Biol Chem 1995; 270: 26690-7.

[34] Rousseau V, Goupille O, Morin N, Barnier J. A new constitutively active brain pak3 isoform displays modified specificities toward rac and cdc42 gtpases. J Biol Chem 2003; 278: 3912-20.

[35] Asrar S, Meng Y, Zhou Z, Todorovski Z, Huang WW, Jia Z. Regulation of hippocampal long-term potentiation by p21-activated protein kinase 1 (PAK1). Neuropharmacology 2009; 56: 73-80.

[36] Penzes P, Beeser A, Chernoff J, et al. Rapid induction of dendritic spine morphogenesis by trans-synaptic Ephrinb-Ephb receptor activation of the Rho-GEF kalirin. Neuron 2003; 37: 263-74.

[37] Chen LY, Rex CS, Casale MS, Gall CM, Lynch G. Changes in synaptic morphology accompany actin signaling during LTP. J Neurosci 2007; 27: 5363-72.

[38] Rex CS, Lin C, Kramár EA, Chen LY, Gall CM, Lynch G. Brainderived neurotrophic factor promotes long-term potentiationrelated cytoskeletal changes in adult hippocampus. J Neurosci 2007; 27: 3017-29.

[39] Burbelo PD, Kozak CA, Finegold AA, Hall A, Pirone DM. Cloning, central nervous system expression and chromosomal mapping of the mouse PAK-1 and PAK-3 genes. Gene 1999; 232: 209-15.

[40] Zhong JL, Banerjee MD, Nikolic M. PAK1 and its 212 phosphorylated form accumulate in neurones and epithelial cells of the developing rodent. Dev Dyn 2003; 228: 121-7.

[41] Hayashi K, Ohshima T, Mikoshiba K. PAK1 is involved in dendrite initiation as a downstream effector of Rac1 in cortical neurons. Mol Cell Neurosci 2002; 20: 579-94.

[42] Hayashi ML, Choi S, Rao BSS, et al. Altered cortical synaptic morphology and impaired memory consolidation in forebrain- specific dominant-negative PAK transgenic mice. Neuron 2004; 42: 773-87.

[43] Zhang H, Webb DJ, Asmussen H, Niu S, Horwitz AF. A GIT1/PIX/Rac/PAK signaling module regulates spine morphogenesis and synapse formation through MLC. J Neurosci 2005; 25 : 3379-88.

[44] Malenka RC, Nicoll RA. Long-term potentiation--a decade of progress? Science 1999; 285: 1870-4.

[45] Matus A. Actin-based plasticity in dendritic spines. Science 2000; 290: 754-8.

[46] Matus A, Brinkhaus H, Wagner U. Actin dynamics in dendritic spines: a form of regulated plasticity at excitatory synapses. Hippocampus 2000; 10: 555-60.

[47] Kelly MT, Yao Y, Sondhi R, Sacktor TC. Actin polymerization regulates the synthesis of PKMzeta in LTP. Neuropharmacology 2007; 52: 41-5.

[48] Sacktor TC, Osten P, Valsamis H, Jiang X, Naik MU, Sublette E. Persistent activation of the zeta isoform of protein kinase $\mathrm{C}$ in the maintenance of long-term potentiation. Proc Natl Acad Sci USA 1993; 90: 8342-6.

[49] Frost JA, Steen H, Shapiro P, et al. Cross-cascade activation of ERKs and ternary complex factors by Rho family proteins. EMBO J 1997; 16: 6426-38.

[50] Coles LC, Shaw PE. PAK1 primes MEK1 for phosphorylation by Raf-1 kinase during cross-cascade activation of the ERK pathway. Oncogene 2002; 21: 2236-44.

[51] Kelleher RJ, Govindarajan A, Tonegawa S. Translational regulatory mechanisms in persistent forms of synaptic plasticity. Neuron 2004; 44: 59-73.

[52] Asrar S, Zhou Z, Ren W, Jia Z. $\mathrm{Ca}^{2+}$ permeable AMPA receptor induced long-term potentiation requires PI3/MAP kinases but not Ca/Cam-dependent kinase II. PLoS ONE 2009; 4: e4339.

[53] English JD, Sweatt JD. A requirement for the mitogen-activated protein kinase cascade in hippocampal long term potentiation. J Biol Chem 1997; 272: 19103-6.

[54] Chelly J, Mandel JL. Monogenic causes of X-linked mental retardation. Nat Rev Genet 2001; 2: 669-80. 
[55] van Galen EJM, Ramakers GJA. Rho proteins, mental retardation and the neurobiological basis of intelligence. Prog Brain Res 2005; 147: 295-317.

[56] Boda B, Alberi S, Nikonenko I, et al. The mental retardation protein PAK3 contributes to synapse formation and plasticity in hippocampus. J Neurosci 2004; 24: 10816-25.

[57] Kreis P, Thévenot E, Rousseau V, Boda B, Muller D, Barnier J. The $\mathrm{p} 21$-activated kinase 3 implicated in mental retardation regulates spine morphogenesis through a Cdc42-dependent pathway. J Biol Chem 2007; 282: 21497-506.

[58] Kandel ER. The molecular biology of memory storage: a dialog between genes and synapses. Biosci Rep 2001; 21: 565-611.

[59] Lonze BE, Ginty DD. Function and regulation of CREB family transcription factors in the nervous system. Neuron 2002; 35: 60523.

[60] Silva AJ. Molecular and cellular cognitive studies of the role of synaptic plasticity in memory. J. Neurobiol 2003; 54: 224-37.

[61] Alarcón JM, Malleret G, Touzani K, et al. Chromatin acetylation, memory, and LTP are impaired in $\mathrm{CBP}+/$ - mice: a model for the cognitive deficit in Rubinstein-Taybi syndrome and its amelioration. Neuron 2004; 42: 947-59.

[62] Korzus E, Rosenfeld MG, Mayford M. CBP histone acetyltransferase activity is a critical component of memory consolidation. Neuron 2004; 42: 961-72.

[63] Graves L, Dalvi A, Lucki I, Blendy JA, Abel T. Behavioral analysis of CREB alphadelta mutation on a B6/129 F1 hybrid background. Hippocampus 2002; 12: 18-26.

[64] Balschun D, Wolfer DP, Gass P, et al. Does cAMP response element-binding protein have a pivotal role in hippocampal synaptic plasticity and hippocampus-dependent memory? J Neurosci 2003; 23: 6304-14.

[65] Leung T, Manser E, Tan L, Lim L. A novel serine/threonine kinase binding the Ras-related RhoA GTPase which translocates the kinase to peripheral membranes. J Biol Chem 1995; 270: 29051-4.

[66] Fujisawa K, Fujita A, Ishizaki T, Saito Y, Narumiya S. Identification of the Rho-binding domain of p160ROCK, a Rho-associated coiled-coil containing protein kinase. J Biol Chem 1996; 271: 23022-8.

[67] Feng J, Ito M, Kureishi Y, et al. Rho-associated kinase of chicken gizzard smooth muscle. J Biol Chem 1999; 274: 3744-52.

[68] Shirao S, Kashiwagi S, Sato M, et al. Sphingosylphosphorylcholine is a novel messenger for Rho-kinase-mediated $\mathrm{Ca}^{2+}$ sensitization in the bovine cerebral artery: unimportant role for protein kinase $\mathrm{C}$. Circ Res 2002; 91: 112-9.

[69] Yoneda A, Multhaupt HAB, Couchman JR. The Rho kinases I and II regulate different aspects of myosin II activity. J Cell Biol 2005; 170: 443-53

[70] Kimura K, Fukata Y, Matsuoka Y, et al. Regulation of the association of adducin with actin filaments by Rho-associated kinase (Rho-kinase) and myosin phosphatase. J Biol Chem 1998; 273: $5542-8$.

[71] Maekawa M, Ishizaki T, Boku S, et al. Signaling from Rho to the actin cytoskeleton through protein kinases ROCK and LIM-kinase. Science 1999; 285: 895-8.

[72] Tashiro A, Yuste R. Regulation of dendritic spine motility and stability by Rac1 and Rho-kinase: evidence for two forms of spine motility. Mol Cell Neurosci 2004; 26: 429-40.

[73] O'Kane EM, Stone TW, Morris BJ. Increased long-term potentiation in the CA1 region of rat hippocampus via modulation of GTPase signalling or inhibition of Rho kinase. Neuropharmacology 2004; 46: 879-87.

[74] Rex CS, Chen LY, Sharma A, et al. Different Rho-GTPasedependent signaling pathways initiate sequential steps in the consolidation of long-term potentiation. J Cell Biol 2009; 186: 85-97.
[75] Lamprecht R, Farb CR, LeDoux JE. Fear memory formation involves p190 RhoGAP and ROCK proteins through a GRB2mediated complex. Neuron 2002; 36: 727-38.

[76] Saitoh A, Yamada M, Yamada M, et al. ROCK inhibition produces anxiety-related behaviors in mice. Psychopharmacology (Berl.) 2006; 188: 1-11.

[77] Zhou Z, Meng Y, Asrar S, Todorovski Z, Jia Z. A critical role of Rho-kinase ROCK2 in the regulation of spine and synaptic function. Neuropharmacology 2009; 56: 81-9.

[78] Hall A. Rho-GTPases and the actin cytoskeleton. Science 1998; 279: $509-14$.

[79] Arber S, Barbayannis FA, Hanser H, et al. Regulation of actin dynamics through phosphorylation of cofilin by LIM-kinase. Nature 1998; 393: 805-9.

[80] Yang N, Higuchi O, Ohashi K, et al. Cofilin phosphorylation by LIM-kinase 1 and its role in Rac-mediated actin reorganization. Nature 1998; 393: 809-12.

[81] Amano T, Kaji N, Ohashi K, Mizuno K. Mitosis-specific activation of LIM motif-containing protein kinase and roles of cofilin phosphorylation and dephosphorylation in mitosis. J Biol Chem 2002; 277: 22093-102.

[82] Vardouli L, Moustakas A, Stournaras C. LIM-kinase 2 and cofilin phosphorylation mediate actin cytoskeleton reorganization induced by transforming growth factor-beta. J Biol Chem 2005; 280: 11448-57.

[83] Foletta VC, Lim MA, Soosairajah J, et al. Direct signaling by the BMP type II receptor via the cytoskeletal regulator LIMK1. J Cell Biol 2003; 162: 1089-98.

[84] Soosairajah J, Maiti S, Wiggan O, et al. Interplay between components of a novel LIM kinase-slingshot phosphatase complex regulates cofilin. EMBO J 2005; 24: 473-86.

[85] Tursun B, Schlüter A, Peters MA, et al. The ubiquitin ligase Rnf6 regulates local LIM kinase 1 levels in axonal growth cones. Genes Dev 2005; 19: 2307-19.

[86] Schratt GM, Tuebing F, Nigh EA, et al. A brain-specific microRNA regulates dendritic spine development. Nature 2006; 439: 283-9.

[87] Meng Y, Zhang Y, Tregoubov V, et al. Abnormal spine morphology and enhanced LTP in LIMK-1 knockout mice. Neuron 2002; 35: 121-33.

[88] Meng Y, Takahashi H, Meng J, et al. Regulation of ADF/cofilin phosphorylation and synaptic function by LIM-kinase. Neuropharmacology 2004; 47: 746-54

[89] Ong WY, Wang XS, Manser E. Differential distribution of alpha and beta isoforms of p21-activated kinase in the monkey cerebral neocortex and hippocampus. Exp Brain Res 2002; 144: 189-99.

[90] Bramham CR. Local protein synthesis, actin dynamics, and LTP consolidation. Curr Opin Neurobiol 2008; 18: 524-31.

[91] Lynch MA. Long-term potentiation and memory. Physiol Rev 2004; 84: 87-136.

[92] Shi Y, Pontrello CG, DeFea KA, Reichardt LF, Ethell IM. Focal adhesion kinase acts downstream of Ephb receptors to maintain mature dendritic spines by regulating cofilin activity. J. Neurosci 2009; 29: 8129-42.

[93] Yang EJ, Yoon J, Min DS, Chung KC. LIM kinase 1 activates cAMP-responsive element-binding protein during the neuronal differentiation of immortalized hippocampal progenitor cells. J Biol Chem 2004; 279: 8903-10.

[94] Jacobs T, Causeret F, Nishimura YV, et al. Localized activation of p21-activated kinase controls neuronal polarity and morphology. J Neurosci 2007; 27: 8604-15.

This is an open access article licensed under the terms of the Creative Commons Attribution Non-Commercial License (http://creativecommons.org/licenses/by-nc/3.0/) which permits unrestricted, non-commercial use, distribution and reproduction in any medium, provided the work is properly cited. 